\title{
Lithium Lens for Focusing Protons on Target in the Fermilab Antiproton Source
}

\author{
F.M. Bieniosek and K. Anderson \\ Fermi National Accelerator Laboratory * \\ P.O. Box 500 \\ Batavia, Illinois 60510
}

\section{Abstract}

A lithium lens will be used to focus the $120 \mathrm{GeV}$ proton beam on the antiproton production target to a spot size of about $0.1 \mathrm{~mm}$, as part of the planned upgrade to the FNAL antiproton source target station. Improved focusing increases antiproton yield and corrects for possible future emittance dilution of the incident proton beam. The lens, with a radius of $3 \mathrm{~mm}$ and length of $8 \mathrm{~cm}$, is expected to operate at a gradient of $2667 \mathrm{~T} / \mathrm{m}$ and a current of $120 \mathrm{kA}$. The lens is similar in design to the antiproton collection lens.

\section{INTRODUCTION}

A lithium lens is a cylindrical current-carrying conductor. If the current density in the lithium is uniform, the strength of the focusing magnetic field is linear with radius, and the device acts as a simple lens on high energy particle beams. Such lenses have been used routinely at FNAL for antiproton collection downstream of the antiproton production target. This paper describes the design and operational parameters of a lithium lens to be installed upstream of the antiproton production target. The upstream (proton) lens design is based on the design of the downstream (collection) lens. In addition, the proton lens design incorporates improvements which may be used in future versions of the collection lens such as improved optical quality and enhanced mechanical design of the beryllium window and titanium cooling jacket.

\section{DESIGN CONSIDERATIONS}

\section{A. Lens Optics}

A short focal length final focus lens minimizes the spot size on target for an incident proton beam. In addition, a short focal length reduces local heating of the beryllium entrance window of the downstream collection lens by the proton beam. The lithium lens was chosen over alternate focusing technologies (e.g., plasma lens, pulsed quadrupoles) because the design of the lens can borrow extensively from experience at FNAL gained in fabricating and operating the antiproton collection lens. For a given magnetic field gradient, the radius

* Operated by the Universities Research Association Under Contract with the U.S. Department of Energy of the lens should be as small as possible to minimize stresses encountered in the cooling jacket which surrounds the lithium core. Experience at FNAL [Ref. 1] and elsewhere [Refs. 2,3] indicates that lithium lenses can be made to operate reliably if the magnetic field at the outer radius of the lens $\left(r_{0}\right)$ is maintained below $10 \mathrm{~T}$. The minimum radius is dictated by the properties of the $120 \mathrm{GeV}$ proton beam and transport line. TRANSPORT [Ref. 4] calculations for a $0.2 \pi \mathrm{mm} \cdot \mathrm{mrad}$ emittance beam with $\Delta \mathrm{p} / \mathrm{p}=0.2 \%(95 \%$ limits $)$ in the transport line retuned for the proton lens yield a half-beam size of $1.7 \mathrm{~mm}$ at the upstream entrance of the lens. Allowing for beam sweep on target, variations in emittance, and drift in beam position, a $3 \mathrm{~mm}$ lens radius should be adequate for efficient beam transport. A lens of $8 \mathrm{~cm}$ length located at the first collimator module of the target station (approximately $168 \mathrm{~cm}$ upstream of the target) and operated at a gradient of $2667 \mathrm{~T} / \mathrm{m}$ would focus the beam to an RMS spot size on target of $\sigma_{x}=\sigma_{y}=0.1 \mathrm{~mm}$. At the design radius, the edge field will be $8 \mathrm{~T}$. To provide flexibility in placement of the lens, the design is based on a maximum operating field of 10 $T$. If the emittance of the proton beam should be larger than expected, it will be possible to maintain the desired spot size by placing the lens closer to the target and operating at a field higher that $8 \mathrm{~T}$.

A pulsed power supply delivers a half-sine wave current to the lens described by $I(t)=I_{0} \cdot \exp (-\alpha t) \cdot \sin (\omega t)(0<\omega t<\pi)$, where $I_{0}$ is the peak current without damping, $\alpha$ is the damping constant, and $\omega$ is the angular frequency determined by the capacitance and inductance of the pulser circuit. The current and magnetic field diffuse into the lithium core on a time scale that depends on the ratio of the skin depth to the lens radius $\delta / r_{0}$ [Ref. 5]. Aberrations in the lens are minimized when the magnetic field approaches a linear radial dependence. Aberrations $\Delta r$ at the focal point may be written as a function of radial position at the lens $r$ as:

$$
\Delta r(r)=\left(\frac{H(r)}{r G}-1\right) \cdot r
$$

where $H(r)$ and $G$, the magnetic field at a given lens radius and the gradient of the linear component of the field, respectively, may be calculated from the analytic solution of Reference 5 . We calculate the RMS value of the aberration $\left\langle\Delta r^{2}\right\rangle^{1 / 2}$ as: 


$$
\left\langle\Delta r^{2}\right\rangle^{I / 2}=\frac{I}{G}\left\langle(H-r G)^{2}\right\rangle^{I / 2}
$$

where

$$
\left\langle(H-r G)^{2}\right\rangle^{I / 2}=\frac{2}{r_{0}^{2}} \int_{0}^{r_{0}}(H-r G)^{2} r \cdot d r
$$

which is solved in Fig. 1 for $\delta / r_{0}=0.6,0.65,0.7$, and 0.75 $\left(\alpha=6760 \mathrm{sec}^{-1}, \omega=41000 \mathrm{sec}^{-1}\right)$.

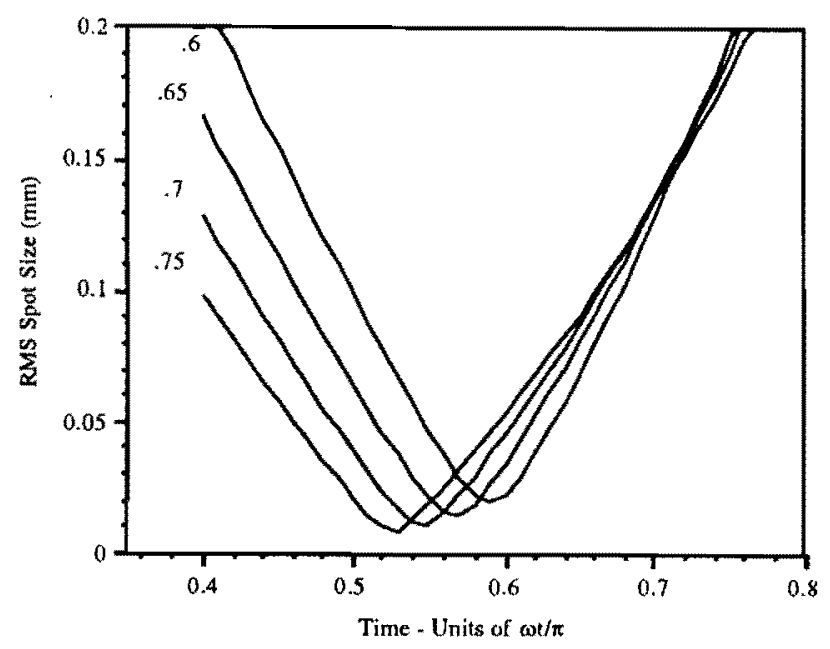

Figure 1. Proton lens field linearity.

This calculation assumes that the incident proton beam is free to wander across the aperture of the lens. The ratio $\delta / r_{0}$ is set at 0.7 to allow for jitter in the trigger circuit of the lens pulser; $\left\langle\Delta r^{2}\right\rangle^{I / 2}$ remains less than $0.02 \mathrm{~mm}$ in radius over a $5 \mu$ s time window.

The ratio $\delta / r_{0}$ changes as the lithium is heated during the pulse. Temperature and conductivity both vary significantly as a function of radius during the pulse; typical curves for the temperature distribution are shown in Fig. 2.

The solution of Ref. 5 neglects the conductivity profile in the lithium; solving the complete coupled equations requires a numerical solution [Ref. 6]. The full solution has not yet been completed in this case; however, if the conductivity profile is parabolic, one can show that the spot size is not significantly degraded, although the timing of minimum spot size may change slightly.

Multiple scattering, which amounts to about $27.5 \mu \mathrm{rad}$, is the dominant contributor to spot size growth from beam interaction with the proton lens. The quadrature sum of all lens contributions, including multiple scattering, finite depth of focus, and optical aberrations, to spot size is about 0.05 $\mathrm{mm}$. This value is smaller than the limiting spot size for the expected emittance of the proton beam delivered to the lens $(0.1 \mathrm{~mm})$. As a result, lens imperfections are not expected to be a significant factor in determining the attainable spot size. The total beam absorption loss is $7.5 \%$ (i.e., $5.8 \%$ in the lithium and $1.7 \%$ in the beryllium windows).

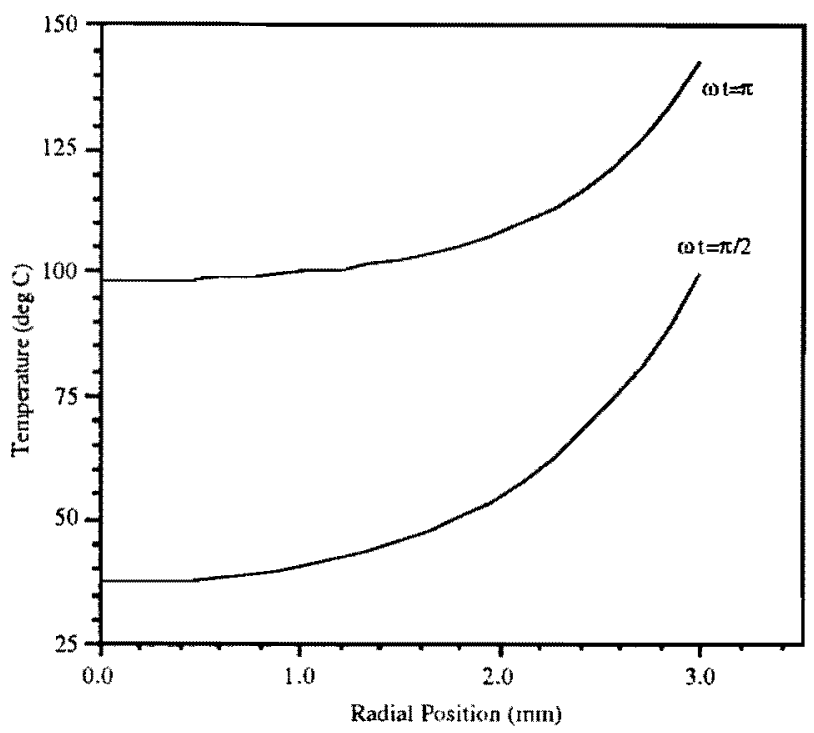

Figure 2. Temperature profile in lithium core at peak current and after pulse during cyclic operation.

\section{B. Mechanical Design}

A cross-section of the proton lens is shown in Fig. 3. The current is introduced to the lithium through 1018 steel, which has relatively low resistivity, adequate strength, and is compatible with liquid lithium.

The cooling jacket is machined from Ti $6 \mathrm{Al}-4 \mathrm{~V}$ ELI billet bar stock. The various pieces of the water circuit septum are joined by electron beam welding. Great care is exercised in preparing and joining the septum components to minimize weld embrittlement and avoid formation of deleterious microstructures. The central core of lithium is constrained by a $1 \mathrm{~mm}$ thick Ti $6 \mathrm{Al}-4 \mathrm{~V}$ conductor tube which provides the path for heat exchange between the lithium core and the cooling water. The thickness of the conductor tube was selected such that it carries only a small fraction (i.e., $<5 \%$ ) of the total current, minimizes the combined stresses in the tube during operation, and minimizes the temperature gradient across the tube wall. Calculations indicate that the largest stress in the cooling jacket occurs just after the pulse and is due primarily to the thernal expansion of the lithium core. The largest radial stress occurs at the inner wall of the conductor tube and is estimated to be $14.7 \mathrm{ksi}$. The highest tangential stress (i.e., maximum principal tensile stress) also occurs at the inner wall of the conductor tube and is calculated to be $52 \mathrm{ksi}$, which is well below the endurance limit of the material for $10^{7}$ cycles. As delineated in Fig. 2, the temperature of the lithium core remains well below the melting point of $186^{\circ} \mathrm{C}$ during cyclic operation. 


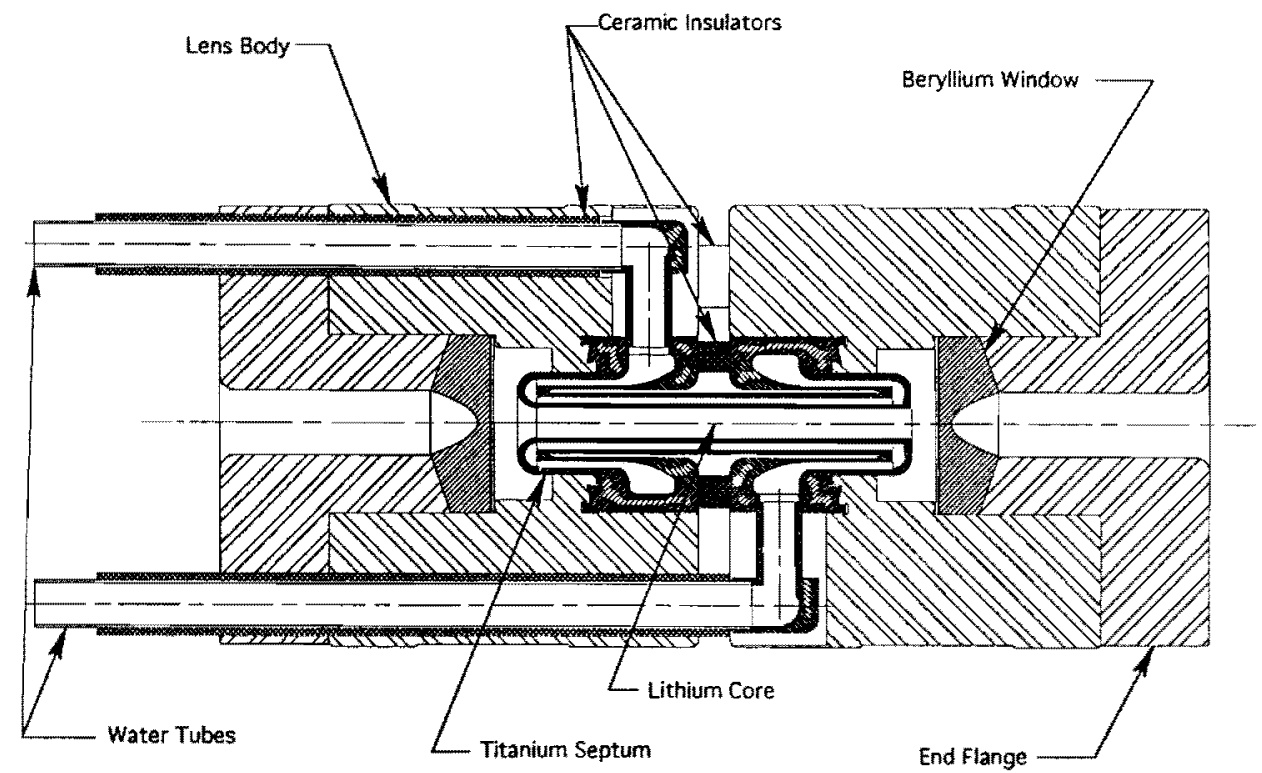

Figure 3. Cross-Sectional View of Proton Lens Assembly

The septum endcaps feature a uniform gradation in crosssection to provide the transition in wall thickness of $1 \mathrm{~mm}$ in the conductor tube to $1.5 \mathrm{~mm}$ thickness in the outer current director base. Such design minimizes stress concentrations due to changing wall thickness and provides adequate strength in the critical endcap region.

As delineated in Fig. 3, the beryllium window features a parabolic profile which minimizes the window thickness and consequently beam absorption while maintaining adequate strength to prevent fracture. Beryllium was selected to minimize beam absorption and multiple scattering which could dilute the effective spot size on target. The minimum window thickness is $3 \mathrm{~mm}$. The peak axial pressure exerted on the window (about $30 \mathrm{ksi}$ ) is primarily due to the sum of axial pressures generated by radially flowing currents in the end region and by Poisson effect loads associated with radial compression of the central lithium core. The inclined boundary of the window serves to minimize the maximum principal tensile stress at the center of the window and results in a more homogeneous state of stress in the window. The window was modeled using the finite element program ANSYS ${ }^{\circledR}$ [Ref. 7]. The resulting equivalent stress in the window is estimated to be below the $10^{7}$ cycle endurance limit for notched specimens of $S 65$ beryllium of $24 \mathrm{ksi}$.

The steel lens bodies are separated by $95 \%$ alumina ceramic standoffs. The lens current will be monitored by a Rogowski coil placed around the center of the lens body in the transformer package. The entire assembly is constrained against axial loading by $8 \mathrm{Ti} 6 \mathrm{Al}-4 \mathrm{~V}$ preloaded fatigue studs.

Lithium will be introduced into the lens in a procedure similar to that used for the collection lens [Ref. 5]. The lithium will be extruded such that a compressive preload of
210 atm will be maintained at ambient conditions to counteract the maximum $z$-pinch at $\omega t=\pi / 2$.

The proton lens body current contact area was designed to accommodate the same transforner package as that used in the $2 \mathrm{~cm}$ diameter collection lens. The total inductance of the secondary pulser circuit is expected to be $30 \mathrm{nH}$; the secondary circuit resistance is $490 \mu \Omega$, of which the lithium central conductor contributes $330 \mu \Omega$. The primary circuit consists of a power supply capacitance of $260 \mu \mathrm{F}$, an equivalent circuit inductance of $2.5 \mu \mathrm{H}$, and an equivalent circuit resistance of 35 $\mathrm{m} \Omega$. For a nominal operating field of $8 \mathrm{~T}$, the charge voltage on the capacitor bank is $\mathrm{V}=2.0 \mathrm{kV}$, the peak primary circuit current is $15 \mathrm{kA}$, and the current pulse is $80 \mu$ s long. The corresponding lens current is $120 \mathrm{kA}$.

\section{REFERENCES}

(1) G. Biallas, et al.. "Power Tests of the Fermilab Lithium Lens for Antiproton Collection". Proceedings of the 12th International Conference on High Energy Accelerators. Batavia, IL. 1983, pp. 591-593.

(2) D.C. Fiander, "Beam Tests of a 2 cin Diameter Lithium Lens", IEEE Transactions on Nuclear Science. Vol. NS-32, 1985. PP, $3063-3065$

(3) B.F. Bayanov, et al.. "A Lithium Lens for Axially Symmetric Focusing of High Energy Particle Beams". Nuclear Instruments and Methods, Vol. 190, p.9, 1981.

(4) D.C. Carey, "High Energy Charged Particle Optics Computer Programs". Nuclear Instruments and Methods, Vol. 187, pP. 97-102, 1981

(5) A.l. Lennox. "The Design Paraneters for a Lithium Lens as Antiproton Collector". IEEE Transactions on Nuclear Science, Vol. NS-30, 1983, pp. 3663-3665.

(6) H. Knoepfel, Pulsed High Magnetic Fields, North-Holland Publishing Company. 1970, p. 88.

(7) ANSYS Engineering Analysis System. Swanson Analysis Systems. Inc., Houston. PA, 1990. 